\title{
HIGHLY POLARIZED ELECTRON BEAMS FOR LINEAR COLLIDERS *
}

\author{
J. E. Clendenin
}

Stanford Linear Accelerator Center, Stanford, CA 94309

\begin{abstract}
The SLC operates with electron beams having a polarization of $\sim 80 \%$. The physics opportunities for future colliders will be greatly enhanced if the beam polarization can be significantly higher. The prospects for achieving electron single-beam polarizations $>95 \%$ are discussed. A complimentary achievement would be to use two-beam polarization.
\end{abstract}

Presented at the

2nd International Workshop on Electron-Electron Interactions at TeV Energies

University of California, Santa Cruz, CA, September 22-24, 1997

*Work supported by Department of Energy contract DE-AC03-76SF00515. 


\title{
HIGHLY POLARIZED ELECTRON BEAMS FOR LINEAR COLLIDERS*
}

\author{
J. E. CLENDENIN \\ Stanford Linear Accelerator Center \\ Stanford University, Stanford, CA 94309
}

\begin{abstract}
The SLC operates with electron beams having a polarization of $\sim 80 \%$. The physics opportunities for future colliders will be greatly enhanced if the beam polarization can be significantly higher. The prospects for achieving electron single-beam polarizations $>95 \%$ are discussed. A complimentary achievement would be to use two-beam polarization.
\end{abstract}

\section{Polarization Requirements}

Polarization will be an important element for particle physics employing future electron linear colliders. At $\sqrt{s} \approx 500 \mathrm{GeV}$, the cross sections for many processes depend on polarization. Within the SM the striking example is that the production of $W^{+} W^{-}$pairs, which provide a major part of the background for many other processes, is nearly suppressed for a right-handed electron beam. Within SUSY, the production of right-handed sleptons and neutralinos dominates for a right-handed beam, whereas left-handed sleptons and charginos dominate for left-handed. ${ }^{1}$

Thus polarization will be very useful for sorting out SUSY signals. In addition, precision measurements of properties of SUSY particles will benefit from the background reduction available with right-handed electron beams. In the rather dramatic example shown in Figure 1, the dominating $W^{+} W^{-}$background associated with smuon pair production using an unpolarized electron beam is nearly eliminated by using a highly polarized beam. Finally, as will be discussed below, polarized beams enhance the luminosity of a collider.

The principal reason for the effectiveness of polarization is that for energies $>m_{z}$, right-handed electrons (left-handed positrons), $e_{R}^{-}\left(e_{L}^{+}\right)$, have no weak interaction whereas left-handed electrons (right-handed positrons), $e_{L}^{-}\left(e_{R}^{+}\right)$, do. Consequently, above the $Z$ mass, $e_{R}^{-}$and $e_{L}^{-}$behave as distinctly different particles as do $e_{R}^{+}$and $e_{L}^{+}$. Wherever cross sections have a strong dependence on polarization, about half the particles in an unpolarized beam are useless. By choosing only the desired particles for an interaction, the luminosity for a given beam intensity is effectively increased.

*Also published as SLAC-PUB-7726, a publication of the Stanford Linear Accelerator Center. Work supported by Department of Energy contract DE-AC03-76SF00515. 


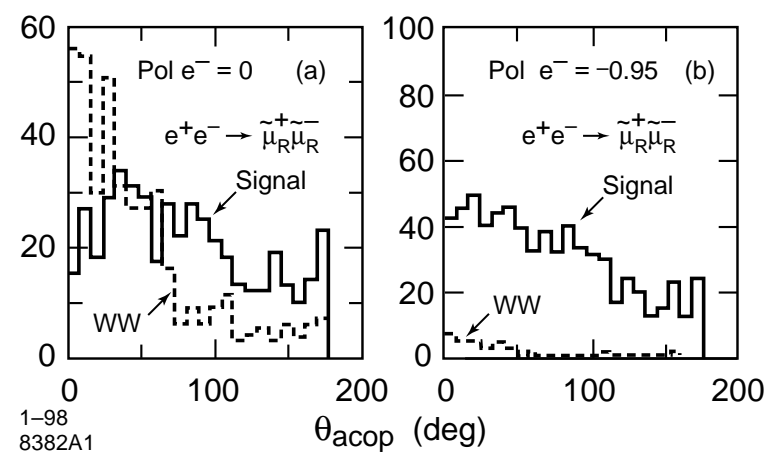

Fig. 1. Examples of acoplanarity distributions for smuon pair production with (a) unpolarized electron beam and (b) a $95 \%$ right-handed polarized beam. Figure from ref. 2.

The polarization for an ensemble of electrons can be defined as

$$
P_{-}=\frac{n\left(e_{L}^{-}\right)-n\left(e_{R}^{-}\right)}{n\left(e_{L}^{-}\right)+n\left(e_{R}^{-}\right)}
$$

and correspondingly for positrons

$$
P_{+}=\frac{n\left(e_{R}^{+}\right)-n\left(e_{L}^{+}\right)}{n\left(e_{R}^{+}\right)+n\left(e_{L}^{+}\right)}
$$

where $n$ indicates the number of particles of a given species. Thus the polarization for left-handed (right-handed) electrons (positrons) is positive.

For future lepton colliders, $\left|P_{-}\right|=80 \%$-which as will be discussed in section 2 is already available-is sufficient for most high-energy physics experiments. A possible exception may be for the study of charginos since their cross sections vary with the handedness of the beam polarization in nearly the same manner as the $W^{+} W^{-}$ background. However, there is no question but that having significantly higher polarization will have an enormous impact on the physics capabilities of a future collider.

\section{Present Capabilities and Limitations}

Polarized electron beams for accelerators were pioneered at SLAC in the mid-1970s. By the 1990s several accelerators including the MIT/Bates linac and the Mainz Microtron (MAMI) were also conducting some physics experiments using polarized beams. Since 1992, all electron beams at SLAC, including the SLC and fixed-target beams, have been polarized. Recently new or revived polarized beams have come online at TJNAF, NIKHEF, and Bonn.

One of the principal factors in the progress of polarized beams has been the semiconductor-photocathode source first introduced to accelerators in 1978. The symmetry for minimum-energy transitions between the valence and conduction bands in III-V semiconductors is very high: equivalent to $j=3 / 2$ symmetry at 
(a)
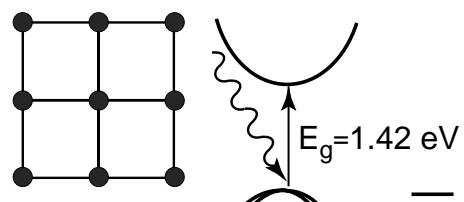

$-1 / 2 \frac{1 / 2}{2}$

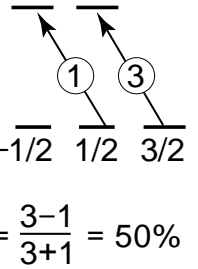

$a=5.654 \AA$

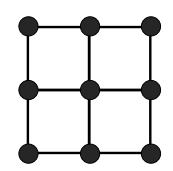

lh

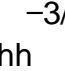

$\Gamma$

$P=\frac{3-1}{3+1}=50 \%$

$\mathrm{GaAs}_{0.72} \mathrm{P}_{0.28}$

$\mathrm{a}=5.596 \AA$

(b)
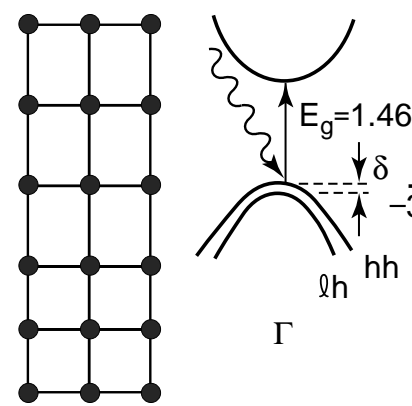

$-\underline{1 / 2} \underline{1 / 2}$

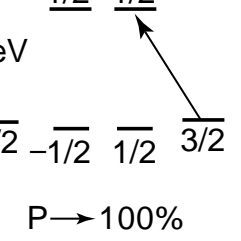

$\Gamma$

\section{Strained GaAs}

(GaAs on

$\left.\mathrm{GaAs}_{0.72} \mathrm{P}_{0.28}\right)$

$1-98$
$8382 A 2$

Fig. 2. Crystal lattice, energy level diagrams at the $\Gamma$ point, and transition probabilities for (a) unstrained GaAs, and (b) strained GaAs on $\mathrm{GaAs}_{0.72} \mathrm{P}_{0.28}$. The constants shown are for room temperature. In (b) the heavy-hole (hh) valence band is separated from the light-hole (lh) at $\Gamma$ by $\delta$. On the right, only transitions for $\sigma^{-}$excitation are shown. The resulting polarizations assume near threshold excitation.

the valence band maximum (VBM) and $1 / 2$ at the conduction band minimum (CBM). Since the transition rate for $m_{j}= \pm 3 / 2 \rightarrow \pm 1 / 2$ is three times that for $\pm 1 / 2 \rightarrow \mp 1 / 2$, absorption of circularly-polarized photons (angular momentum of unity) with energy near the band gap will preferentially populate one spin state in the conduction band. Fortunately the band gap of most III-V semiconductors is near that of several readily available high-power laser systems.

Semiconductor photocathodes having nearly degenerate p-doping can be prepared to have a negative electron affinity (NEA) surface-which produces a high quantum efficiency (QE, defined as ratio of number of extracted electrons to number of incident photons) - by an activation process that includes preparing an atomically clean surface in ultra-high vacuum conditions, then treating the surface with 


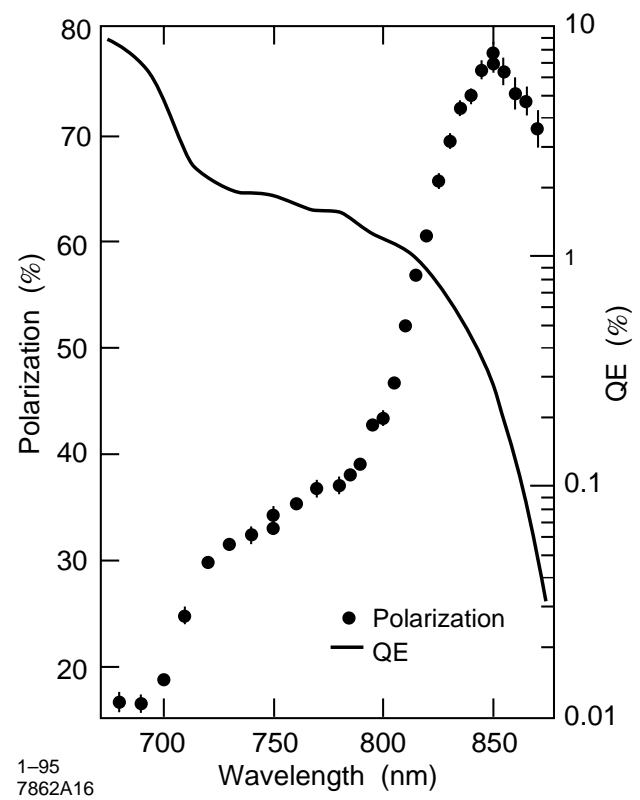

Fig. 3. QE and polarization spectra for the SLAC GaAs on $\mathrm{GaAs}_{0.69} P_{0.31}$ strained-layer photocathode used for SLC in 1994. The measurements were made at room temperature and low voltage.

one (or up to a few) monolayers of Cs and an oxide (typically oxygen or fluorine). The problems of obtaining an initially high QE and maintaining this QE over time have been mostly solved. ${ }^{3}$

Because of the valence-band degeneracy at the $\Gamma$ point of the $m_{j}= \pm 3 / 2$ (heavy hole, hh) with the $m_{j}= \pm 1 / 2$ (light hole, lh) states, the extracted electrons for an unstrained III-V semiconductor have a theoretical polarization of $50 \%$ which is rarely achieved in practice. Most accelerator sources have been limited to 25-35\% polarization. However, in the early 1990 s, a practical means was found to break this degeneracy. Strain is introduced in the crystal lattice by growing a thin epitaxial layer on a substrate having a different lattice constant along with a larger band gap. The lattice mismatch results in a distortion of the lattice of the epitaxial layer, the practical effects of which extend for $>100 \mathrm{~nm}$. The strained lattice and corresponding energy levels are shown in Figure $2 \mathrm{~b}$ and can be compared with the unstrained case in Figure 2a. For a lattice mismatch on the order of 1\%, the energy splitting can be $\sim 50 \mathrm{meV}$. If the excitation laser is tuned to promote electrons to the conduction band from the valence-band higher-energy state only, then the theoretical polarization of the conduction band electrons is raised to $100 \%$. In practice values of $75-85 \%$ are achieved at SLAC. To utilize the strained-layer cathodes for high polarization, the laser must be tuned very close to the band-gap energy where the optical absorption rate is rapidly dropping, requiring a considerable increase in laser energy. Likewise, since the thickness of the epilayer, $d$, is forced to be 
much less than the optical absorption depth, $L_{p}$, the charge arriving at the cathode surface for a given number of incident photons is much reduced. Fortunately, however, there appears to be no diminution in the electron escape probability from the surface-either initially after activation or with respect to the fall off with timeso that given a suitably powerful laser, high intensity accelerator beams are quite achievable as demonstrated by the SLAC beams.

The polarization and QE spectra for one of the 100-nm strained layer photocathodes (GaAs/GaAsP) used for the SLC are shown in Figure 3. In fact, the SLC polarized beam already meets most of the requirements for a future collider with the exception that there are only two widely-spaced SLC bunches of $8 \mathrm{nC}$ each whereas most collider designs require about 100 closely-spaced bunches each with $\sim 1 \mathrm{nC}$ of charge. The latter is a major challenge since it has been found that there is a limit to the current density that can be extracted from a GaAs cathode. This limit can be well below the gun space-charge limit. The cathode charge limit is related to the trapping of electrons in surface states during the extraction process, resulting in the rapid growth in the surface potential barrier. The emission is then limited-i.e., the photocurrent saturates for increasing laser power-by the rate at which the surface charge can be neutralized, which is a process that depends among other things on dopant density, crystal quality, temperature, and on the degree of success of the surface activation. A more extensive discussion of the cathode charge limit can be found in ref. 4 .

\section{Prospects for Significantly Higher Electron Polarization ${ }^{a}$}

The limitations in polarization and yield for semiconductor photocathodes can be understood in the framework of the three-step model ${ }^{5}$ illustrated in Figure 4: (1) absorption of the photons in the bulk material resulting in promotion of electrons to the conduction band, (2) transport of the conduction band electrons to the surface, and (3) escape of the surface electrons to vacuum. For GaAs at room temperature, $L_{p}=\alpha^{-1}$ is typically on the order of $1 \mu \mathrm{m}$, where $\alpha$ is the optical absorption coefficient. The transport process is well described by diffusion theory. One reason for the high QE of bulk GaAs is that the diffusion length, $L_{D}$, is typically well matched to $L_{p}$. Here $L_{D}=\sqrt{D \tau}$, where $D$ is the diffusion constant and $\tau$ is the electronic lifetime in the bulk. Finally the surface escape probability is greatly enhanced by having an NEA surface.

The three-step model describes the QE spectrum quite well. In addition, the depolarization mechanisms associated with each of the three steps can be identified. The incident light is here assumed to be $100 \%$ circularly polarized. The depolarization in the first step is primarily associated with inadequate band splitting combined with the expected smearing of the band edges. During the second step, the electron spin has the opportunity to relax prior to escaping into the band bending region (BBR). As will be discussed below, the depolarization mechanisms in the final step

${ }^{a}$ Section 3 is inspired by a similar but unpublished analysis made by A. Subashiev (St. Petersburg Technical University). 


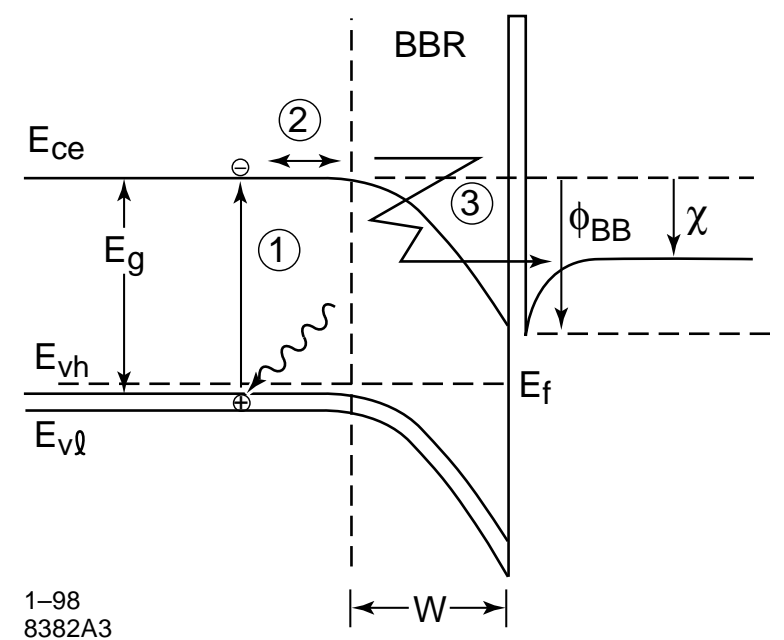

Fig. 4. Schematic energy band diagram at $\Gamma$ near the surface for strained GaAs illustrating the three-step emission process where step 1 is absorption of a photon creating an electron-hole pair, step 2 is escape of the conduction band electron into the band bending region (BBR), and step 3 is the emission of the electron to vacuum. $\mathrm{E}_{c e}, \mathrm{E}_{v h}$, and $\mathrm{E}_{v l}$ are conduction band minimum and valence band maximum for heavy hole and light hole energies in bulk respectively. $\mathrm{E}_{g}$ is the gap energy, $F_{e}$ the fermi energy, $\chi$ the electron affinity, and $W$ and $\phi_{B B}$ the width and depth of the $\mathrm{BBR}$ respectively.

are presently not well understood.

The polarization, $P$, as well as $\mathrm{QE}$ for the extracted electrons as derived using the three-step model can be linearized ${ }^{6}$ if $d<<L_{p}, L_{D}$, or $L_{s}$, where $L_{s}$ is a distance related to the spin relaxation time in the bulk, $\tau_{s}$, by $L_{S}=\sqrt{D \tau_{S}}$. The linearized $\mathrm{QE}$ is given by

$$
Q E=(1-R) \alpha d B_{N}
$$

where $\mathrm{R}$ is the optical reflection coefficient at the surface and $B_{N}$ is the electron escape probability from the BBR.

$P$ can be expressed as the product of three factors, one for each of the three steps:

$$
P=P_{1} P_{2} P_{3}
$$

where $P_{1}$ is the polarization upon initial excitation to the conduction band and $P_{2}\left(P_{3}\right)$ is the probability of escape from the active layer (BBR) without loss of polarization.

$P_{1}$ depends on two factors: the hh-lh band splitting, $\delta$, at the $\Gamma$ point, and any variations in the band edges. For strained-layer cathodes, the strain is perfectly maintained only up to a critical thickness, $d_{c}$, which is typically about $10 \mathrm{~nm}$. Beyond the critical thickness, the strain is expected to decrease at a rate depending somewhat on the lattice defect density, which in turn depends on the fabrication 
details for a specific crystal. Thus the effective $\delta$ can be increased by using a small value of $d$ and by careful fabrication techniques. One of the enticing features of a strained superlattice is that the thickness of the individual quantum wells and barriers are each much smaller than $d_{c}$. For highly doped materials at room temperature, the variations in the band edges are dominated by band tailing, which is the spreading of the band edge due to the random potential fluctuations associated with the ionized acceptors (for p-doped material). The band tailing can be characterized by a band tailing parameter, $\gamma$. Comparison ${ }^{7}$ of calculated values of $\gamma$ with experimental photoluminescence data gives a value of $\gamma \approx 20 \mathrm{meV}$ for a dopant density of $5 \times 10^{18} \mathrm{~cm}^{-3}$. Broadly speaking, $\gamma$ decreases (increases) by a factor of 2 for a decade decrease (increase) in dopant density, i.e., $\gamma \propto N_{a}^{1 / 3}$. The initial polarization of the electrons in the conduction band, $P_{1}$, can be expressed as

$$
P_{1}=1-\delta P_{1}
$$

where $\delta P_{1}$ is the initial depolarization given by

$$
\delta P_{1} \approx e^{-\delta / \gamma} .
$$

Assuming an average value of $\delta=50 \mathrm{meV}$, the initial polarization for a dopant concentration of $5 \times 10^{18} \mathrm{~cm}^{-3}$ is $P_{1} \approx 0.92$. $P_{1}$ can presumably be increased to unity by a combination of larger $\delta$ and smaller $\gamma$.

$P_{2}$ is generally affected by two depolarization components, viz., depolarization during thermalization to the bottom of the conduction band, and depolarization during diffusion to the surface. These two components are in general interdependent for thin active layers since a high proportion of the electrons would escape to the BBR before complete thermalization. However, for excitation at threshold, which is necessary for high polarization, only the second component applies. For nearly degenerate p-doped materials at room temperature, spin relaxation during step 2 is dominated ${ }^{8}$ by the exchange interaction between conduction band electrons and bound holes, known after the authors who first described it as the BAP mechanism. ${ }^{9}$ $P_{2}$ is given in general by

$$
P_{2}=\frac{\tau}{\tau+\tau_{s}} .
$$

Since

$$
\tau=\frac{d}{S_{1}+S_{0}}
$$

where $S_{1}\left(S_{0}\right)$ is the heterointerface (surface) recombination velocity and assuming $S_{1}<<S_{o}$, the expression for $P_{2}$ simplifies to

$$
P_{2} \approx 1-\delta P_{2}=1-\frac{d}{S_{o} \tau_{s}}
$$


for thin active layers. Typical values are $S_{0}=2 \times 10^{6} \mathrm{~cm} / \mathrm{s}$ and $\tau_{s}=5 \times 10^{-11}$ $\mathrm{s}$, where a dopant density of $5 \times 10^{18} \mathrm{~cm}^{-3}$ is assumed. Therefore one expects $S_{o} \tau_{s} \approx 10^{3} \mathrm{~nm}$, which is consistent with the experimental value derived from the slope of $P$ vs. $d$ for thin active layers. ${ }^{6}$

Since for the BAP mechanism, $\tau_{s}$ varies inversely with $N_{a}, \delta P_{2}$ can be essentially eliminated if $N_{a}$ is reduced - at least in the bulk-by an order of magnitude. One could also reduce $d$ to $<100 \mathrm{~nm}$, but at the expense of decreasing the QE.

Polarization losses in the BBR determine the factor $P_{3}$. It is fairly well established ${ }^{10}$ that electrons remain in the BBR for relatively long periods, losing energy by emission of optical phonons before being emitted to vacuum. The BAP mechanism is not applicable in the BBR because of hole depletion. Thus most of the spin relaxation in the BBR is likely due to the diffusion of the spin precession vector - a process known as the DP mechanism ${ }^{11}$ - which is quite sensitive to the K.E. More recently, it has been shown that for thin active layers excited at threshold, the energy distribution of the electrons emitted to vacuum consists of a broad plateau extending in energy from just below the CBM in bulk to the vacuum level. ${ }^{12}$ The high-energy edge of this distribution follows the excitation energy below threshold indicating that the tails of the conduction band play a significant role in the energy distribution of the escaping electrons. These electrons have an energy-resolved polarization that at first decreases gradually with decreasing energy, then levels off. ${ }^{13}$ This relatively abrupt leveling off of the spin relaxation rate at low K.E. has been associated with the electrons becoming localized in fluctuation states associated with the fluctuation potential generated by the random impurity distribution in the BBR and at the surface. ${ }^{14}$ The energy separating localized from delocalized electron states is known as the percolation level (PL).

To minimize the depolarization in the BBR, the K.E. must be kept low. This is done if the excitation energy is near the band gap, as is necessary for producing highly polarized electrons from strained-layer cathodes, and if the PL is high. The PL can be made high by using a high dopant density in the BBR which shifts all the states to higher energy while simultaneously increasing the NEA. ${ }^{7}$ It turns out that high dopant density in the BBR is the prescription for raising the saturated current as well. Differential doping of the BBR and bulk is easily accomplished using either MBE or MOCVD growth techniques. Fortunately the heat cleaning $\left(600^{\circ} \mathrm{C}\right.$ for 1 hour) typically employed to prepare NEA surfaces seems not to result in rapid diffusion of the higher density dopant. ${ }^{15}$

The polarization is known to increase with decreasing QE, i.e., as the vacuum level increases, the electrons that have undergone simultaneous energy and spin relaxation in the BBR are progressively blocked. At the lowest QE the polarization should be equal to that of the electrons when they initially enter the BBR. For the SLAC polarized electron source, the polarization rises from 0.75 to about 0.85 as the QE decreases over one decade. ${ }^{3}$ One would choose to operate with such low QE when sufficient laser power is available except for the cathode charge limit, which unfortunately decreases the saturated current level in proportion to the decreasing 
Table 1. Spin Relaxation Factors.

\begin{tabular}{lll}
\hline Factor & Present Value & Improved Value \\
$\delta \mathrm{P}_{1}$ & 0.06 & 0 \\
$\delta \mathrm{P}_{2}$ & 0.10 & 0 \\
$\delta \mathrm{P}_{3}$ & 0.05 & 0.03 \\
\hline
\end{tabular}

QE. The higher polarization measured at SLAC presumably corresponds to the case where the electrons that escape to vacuum have undergone no spin relaxation in the BBR. Extrapolating this value to zero thickness (see earlier) gives a value of $P_{1}=0.94$, which is slightly higher than estimated earlier.

The spin-relaxation factors associated with each step are summarized in Table 1. The improved value represents what should be achievable based on the analysis in this section.

Thus it appears one should be able to achieve an electron polarization of $P \geq$ 0.95 from a properly designed and fabricated strained-layer or strained-superlattice photocathode.

\section{Polarized Positrons}

Polarized positrons are in general desirable for an $\mathrm{e}^{+} \mathrm{e}^{-}$collider since they enhance the luminosity in a manner similar to that of polarized electrons. Polarized positrons can be produced by pair production in a conversion target using highenergy polarized gammas derived either from a high-energy electron beam ( $\mathrm{E}>150$ $\mathrm{GeV}$ ) in a long helical undulator or from an intense polarized laser beam Compton back-scattered from a relatively low-energy electron beam $(\mathrm{E}<10 \mathrm{GeV}){ }^{16}$

For $\mathrm{e}^{+} \mathrm{e}^{-}$colliders in which both beams are polarized, a generalized polarization, $\mathrm{P}_{g}$, can be defined: ${ }^{17}$

$$
P_{g}=\frac{n\left(e_{L}^{-}\right) n\left(e_{R}^{+}\right)-n\left(e_{R}^{-}\right) n\left(e_{L}^{+}\right)}{n\left(e_{L}^{-}\right) n\left(e_{R}^{+}\right)+n\left(e_{R}^{-}\right) n\left(e_{L}^{+}\right)}=\frac{P_{-}+P_{+}}{1+P_{-} P_{+}}
$$

Assuming a positron polarization of $0.5, \mathrm{P}_{g}$ would be $0.93(0.97)$ for electron polarization of $0.8(0.9)$. Thus for a less than $100 \%$ polarized electron beam, a polarized positron beam would be very powerful complimentary tool for exploring many highenergy physics processes. ${ }^{18}$

\section{Conclusions}

From the discussion above, the prescription for increasing the electron polarization to $\geq 95 \%$ is fairly clear: a thin, strained, active layer (either a single strained-layer or a superlattice) with large $\delta$ that has doping concentration in bulk of $\leq 10^{18} \mathrm{~cm}^{-3}$ and in the BBR of $\geq 5 \times 10^{18} \mathrm{~cm}^{-3}$, carefully fabricated on a high-quality substrate. The research to produce such a cathode, including the investigation of many relevant details not discussed here, is underway at SLAC and other institutions. 


\section{Acknowledgments}

Useful discussion with M. Peskin (SLAC) and A. Subashiev (St. Petersburg Technical University) are gratefully acknowledged.

\section{References}

1. M. Danielson et al., in New Directions for High-Energy Physics, Proceedings of the 1996 DPF/DPB Summer Study on High-Energy Physics, June 25-July 12, 1996, Snowmass, CO, eds. D.G. Cassel et al. (SLAC, Stanford, 1997), p. 720.

2. K. Fujii, in Workshop on Physics and Experiments with Linear Colliders, MoriokaAppi, Iwate, JP, September 8-12, 1995, eds. A. Miyamoto et al. (World Scientific, Singapore, 1996), p. 283.

3. R. Alley et al., Nucl. Instrum. and Meth. A 365 (1995) 1.

4. H. Tang et al., in Proceedings of the Fourth European Particle Accelerator Conference, June 27-July 1, 1994, London, UK, eds. V. Suller et al. (World Scientific, Singapore, 1994), p. 46.

5. W.E. Spicer, Phys. Rev. 112 (1958) 114.

6. B.D. Oskotskij et al., Phys. Low-Dim. Struct. 1/2 (1997) 77.

7. A.V. Subashiev, St. Petersburg Technical University, private communication (1997).

8. G. Fishman and G. Lampel, Phys. Rev. B 16 (1977) 820.

9. G.L. Bir, A.G. Aronov, and G.E. Pikus, Sov. Phys. JETP 42 (1976) 705.

10. J. Kirschner et al., Appl. Phys. A 30 (1983) 177.

11. M.I. D'Yakonov and V.I. Perel', Sov. Phys. JETP 33 (1971) 1053.

12. A.S. Terekhov and D.A. Orlov, SPIE 2550 (1995) 157.

13. H.-J. Drouhin et al., Phys. Rev. B 31 (1985) 3872.

14. L.G. Gerchikov et al., in SPIN96, Proceedings of the 12th International Symposium on High-Energy Spin Physics, Amsterdam, NL, September 10-14, 1996, eds. C.W. de Jager et al. (World Scientific, Singapore, 1997), p. 746.

15. G. Mulhollan, SLAC, private communication (1997).

16. See the Proceedings of the Workshop on New Kinds of Positron Sources for Linear Colliders, March 4-7, 1997, SLAC, eds. J. Clendenin and R. Nixon, SLAC-R-502 (1997), $435 \mathrm{pp}$. Production of polarized positrons by impinging $50 \mathrm{MeV}$ polarized electrons on a thin target has also been described, but the yield as presently estimated is too low for a collider: see E.G. Bessonov and A.A. Mikhailichenko, in Proceedings of the Fifth European Particle Accelerator Conference, June 10-14, 1996, Sitges, SP, eds. S. Myers et al., (IOP Publishing, Bristol, 1996), p. 1516; also A.P. Potylitsin, Nucl. Instrum. and Meth. A 398 (1997) 395.

17. K. Flöttmann, DESY-95-064 (1995).

18. T. Omori in Proceedings of the Workshop on New Kinds of Positron Sources for Linear Colliders, March 4-7, 1997, SLAC, eds. J. Clendenin and R. Nixon, SLAC-R-502 (1997), p. 285. 